\title{
Tunnel and Subsurface Void Detection and Range to Target Measurement
}

\section{IEEE International Conference on Technologies for Homeland Security}

\author{
Phillip B. West
}

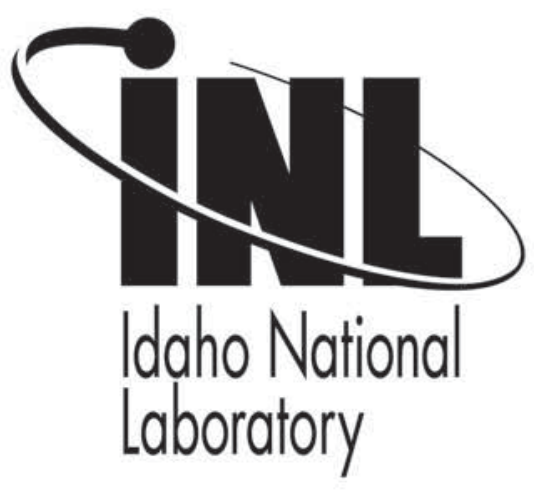

This is a preprint of a paper intended for publication in a journal or proceedings. Since changes may be made before publication, this preprint should not be cited or reproduced without permission of the author. This document was prepared as an account of work sponsored by an agency of the United States Government. Neither the United States Government nor any agency thereof, or any of their employees, makes any warranty, expressed or implied, or assumes any legal liability or responsibility for any third party's use, or the results of such use, of any information, apparatus, product or process disclosed in this report, or represents that its use by such third party would not infringe privately owned rights. The views expressed in this paper are not necessarily those of the United States Government or the sponsoring agency. 


\title{
Tunnel and Subsurface Void Detection and Range to Target Measurement
}

\author{
Phillip B. West \\ Idaho National Laboratory \\ PO Box 1625 \\ Idaho Falls, ID 83415-3730 \\ 208-526-1538 \\ phillip.west@inl.gov
}

\begin{abstract}
Engineers and technicians at the Idaho National Laboratory invented, designed, built and tested a device capable of detecting and measuring the distance to an underground void, or tunnel. Preliminary tests demonstrated positive detection of, and range to, a void thru as much as 30 meters of top-soil earth. Device uses acoustic driving point impedance principles pioneered by the Lab. for well-bore physical properties logging. Data receipts recorded by the device indicates constructivedestructive interference patterns characteristic of acoustic wave reflection from a downward step-change in impedance. Prototype tests demonstrated that interference patterns in receipt waves could depict the patterns indicative of specific distances. A tool with this capability can quickly (in seconds) indicate the presence and depth/distance of a void or tunnel. Using such a device, border security and military personnel can identify threats of intrusion or weapons caches in most all soil conditions including moist and rocky.
\end{abstract}

\section{INTRODUCTION}

The Idaho National Laboratory (INL) Fossil Energy Technology department, in an analytical study, employed wave-equation mathematics describing the "driving point impedance" assay concept. This was done to develop a technology for subsurface geophysical assays. The development included efforts to create the Orbital VibratorPhysical Properties Logger (OV-PPL) and this serves as the basis for this forward scanning, void detecting, hand-held sensor titled; Look Ahead Sensor (LAS). An orbital vibrator is best described as an out-of-balance motor in a shell. OVs have been used for years in seismic surveys as an acoustic source which transmits to remote receivers. The INL developed OV-PPL however, requires no additional components. Once lowered into a fluid-filled (e.g., water, oil) well the device is operated at various frequencies. The OV-PPL produces sufficiently fast vibrations that earthen properties are measured because the acoustic energy passes through the water into the surroundings as if it where directly coupled.

The OV-PPL incorporates two orthogonal, internallymounted motion detectors in the form of geophones that provide motion data. ${ }^{i}$ The recorded orbital motion of the device as it tries to orbit while restrained by the fluid coupling to the earth, characterizes the transmission of the acoustic energy. This characterization, resulting from the radial transmission and sometimes returning reflections of acoustic energy, is indicative of the surrounding media's energy absorbing properties or "dissipative impedance". By evaluating the data receipt, properties of the earth surrounding the well, such as rock types, can be measured, and the presence of an anomaly, such as an underground crack or void, can also be detected.

For "looking ahead", the earth-borne acoustic energy and subsequent measurements are made only in the "forward" direction. The resulting signal profile resembles a cone with the tool at the apex. Consequently, the intended objective of the new tool is best characterized as a "look-ahead" not a "look-around" sensor. 


\section{LOOK AHEAD TECHNOLOGY}

The desire for forward looking, properties detection originated with the need to know what was ahead of an oil/gas drill string. Environmental and cost risks are imposed by drilling blindly forward. To know earth conditions ahead of the drill string, a forward looking sensor was proposed. The concept proposed a device similar to the OV-PPL, except to create a axial signal two synchronized counter rotating eccentrically weighted wheels are used. However, for a drill string mounted application the device would be very constrained in size and geometry.

A DOE funded project sought to enable technologies that could detect subsurface voids from a user's position. The device could ostensibly be of any size and shape and could be directly coupled to the earth. The INL conceived a handheld tool that would incorporate the OV-PPL philosophy but in a forward looking configuration. The INL team developed the concept to an actual tool. The resulting Look Ahead Sensor (LAS) is: 0.58 meter wide by 0.35 meter long by 0.12 meter deep (23" x 14" x 5"). It has a $150 \mathrm{~mm}$ (6") diameter earth contact pad, and weighs approximately $15 \mathrm{~kg}$ (32 lbs), including its onboard lithium battery pack. (see Figure 1) The complete system consists of only the LAS and a laptop computer.

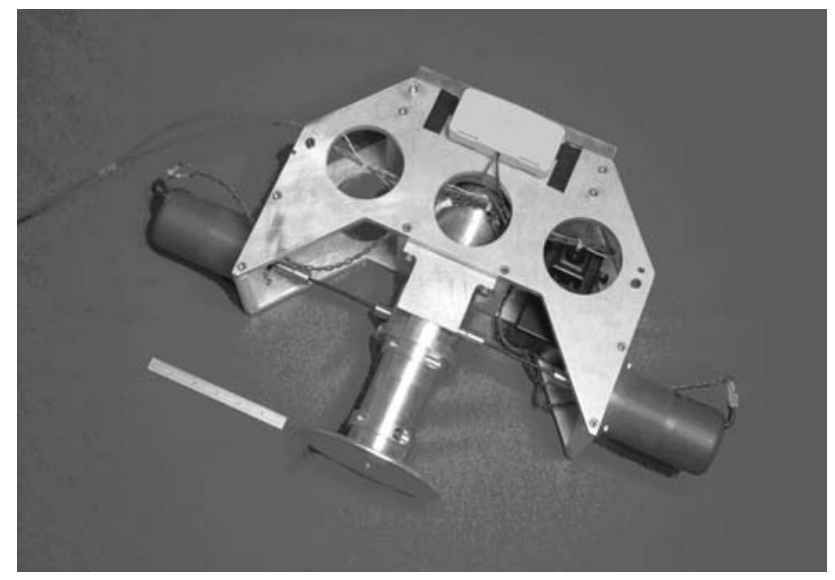

Figure 1. The 2007 Configuration of the Look-Ahead Sensor (LAS), (with 6 in. scale)

The original design goal was a void detection range from 2.5 to 15 meters. However, tests conducted did not include measurements for targets less than 3.75 meters from the device because the shorter range would impose potentially damaging stress on the tool. To mitigate this however, the LAS is configured to allow for in-field changes to adjust the maximum force produced by the tool. The addition of weights that partially balance the imbalance provides this feature. The peak force needs to be variable because the force required for reliable measurement is proportional to distance to be surveyed. Distant voids require stronger but lower frequency vibration force to ensure the signal traverses the desired distance. Detection of less-distant voids requires less force, but higher frequency. With a fixed eccentric mass value, the higher frequencies not only produce forces higher than needed, but higher than can be handled by a human operator.

Regardless of the design features to allow the field installation of different balance-weights to reduced the maximum force, we settled on one setting that appeared to provide a suitable range. The design also includes a pair of jointed drive shafts connecting the motor to the out-ofbalance counter-rotating weights. These components were used to ensure that undesirable dynamic loads neither degraded data nor impeded motor function.

\section{Theory of the Look-Ahead Sensor}

The look-ahead sensor generates its own acoustic energy to transmit into the earth. The character of the transmission and the effect on subsequent transmissions caused by reflections, are used to indicate the presence and distance to a void. The vibrations are actively induced in the ground in the $\sim 60 \mathrm{~Hz}$ to $\sim 200 \mathrm{~Hz}$ range. These vibrations typically propagate through the ground with some attenuation as well as geometric dispersion. Furthermore, if the acoustic energy encounters a change in media, transmission characteristics change, and a reflection occurs. These characteristics are affected by the frequency of the signal. The onboard sensors are capable of detecting the changes in the response of the earth. When no voids are present, the change in response to the change in frequency is detected and represented as a smooth progression from the previous frequency. This progression follows the power function imposed by the force being proportional to the square of the frequency. However, if a void exists, then at particular frequencies the vibration can establish a standing wave between the void location and the device.

When these resonance frequencies occur the onboard geophone detects a significant change in signal amplitude when compared to a no-void condition and this indicates a void. When the change is higher amplitude, constructive interference is occurring. This happens when the void is at $1 / 4$ wavelength distant or any harmonics there after. Because a void represents a downward step change in impedance the reflection is reverse sign or $1 / 2$ wave out of phase. So for constructive interference the signal, traveling there and back must travel a total of $1 / 2$ wavelength. At certain frequencies however the returning wave energy is out of phase with the LAS's outgoing signal. This destructive interference is the result of a reflection condition poised at $3 / 4$ wavelength distant and subsequent harmonics.

Consequently, the distance to the void can be calculated using:

distance $=1 / 4$ wavelength 
Because the speed of sound " $C$ " in the earth can be approximated as $10,000 \mathrm{ft} / \mathrm{sec}$, the wavelength can be estimated from the resonance frequency.

The calculated distance is

$1 / 4$ Wavelength $=1 / 4 \times C /$ frequency for the first (lowest) frequency where constructive interference occurs. Similarly the subsequent harmonics of constructive and destructive interference can be found, and identified. The distance to the void and the presence of the void are dependent only on the acoustic wave speed in the earth. Although the speed of sound in the earth is not uniform, for reasonable accuracies, $10,000 \mathrm{ft} / \mathrm{sec}$ is sufficient.

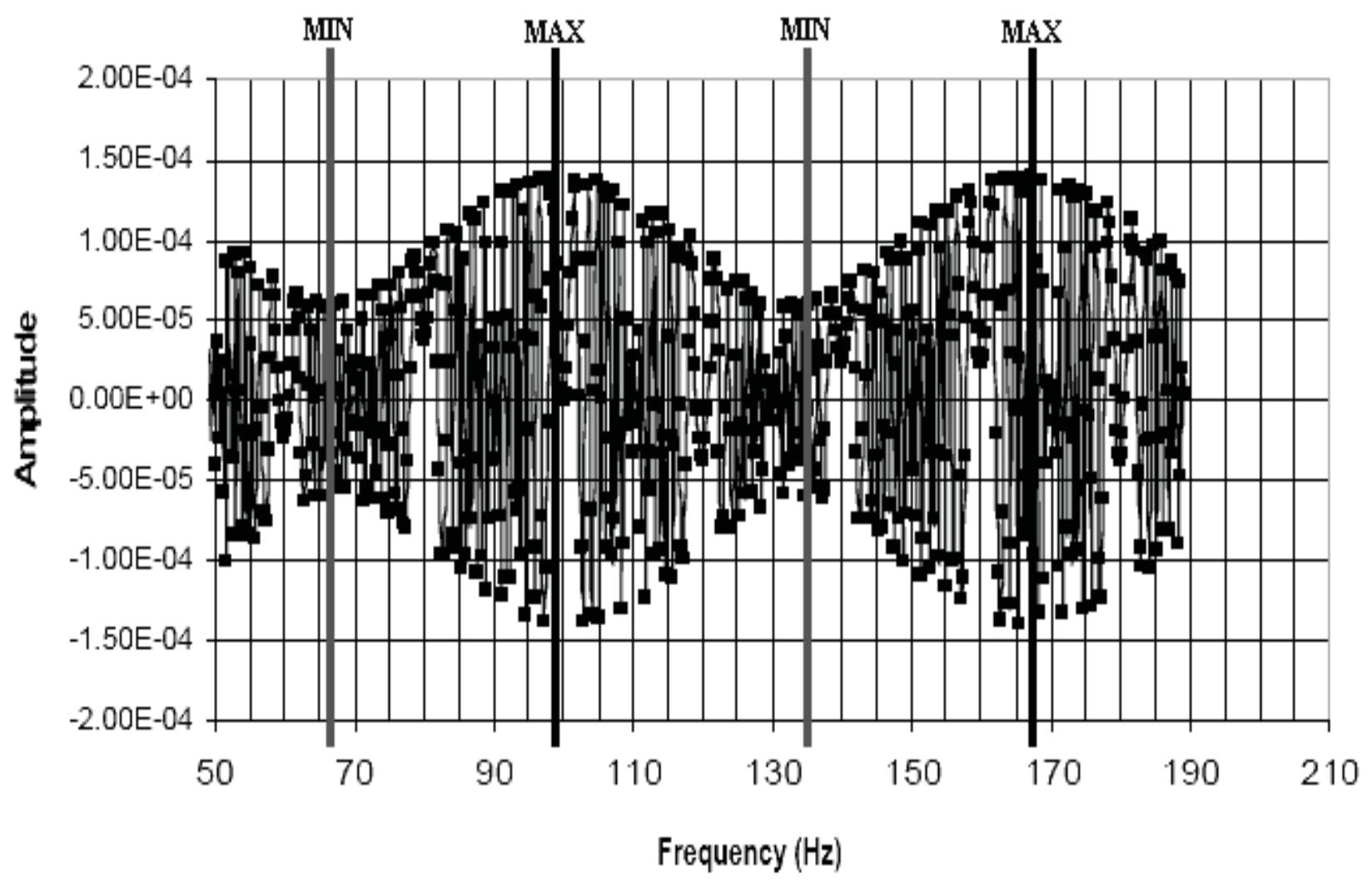

Figure 2. Math Model for Simulated Void (23 meters) and $\mathrm{C}=10000 \mathrm{ft} / \mathrm{sec} .1^{\text {st }}$ and $3^{\text {rd }}$ lines are destructive (MIN) interference, $2^{\text {nd }}$ and 4 th lines are constructive (MAX) inter ference. 


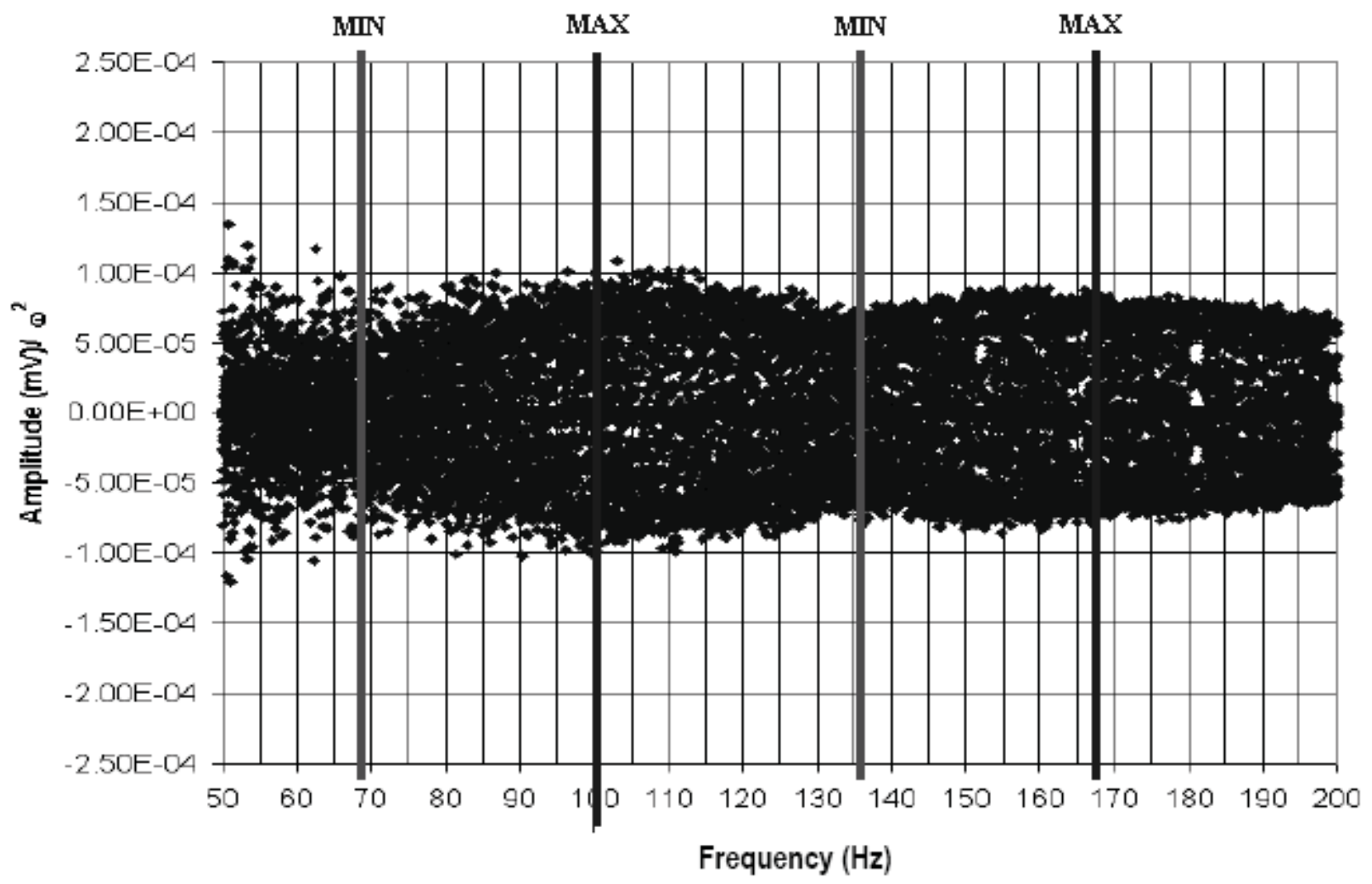

Figure 3. Rye Grass Flats "Processed" Data - Void (23 meters)

Therefore, the look-ahead sensor can be expected to perform well in all packed earth scenarios. Figure 2 is a mathematic depiction of theoretical constructive-destructive interference pattern using $10,000 \mathrm{ft} / \mathrm{min}$ wave speed, $77 \mathrm{ft}(23 \mathrm{~m})$ distance. This math model however assumes constant force and attenuation vs frequency. Early testing produced data, that when normalized by dividing amplitude by frequency squared to account for force rising with frequency squared (Figure 3), correlated well with the math model.

\section{TESTING AT THE INL}

A test range was developed for evaluating the LAS; the Rye Grass Flats (RGF) area. A trench was excavated at an acute angle to an existing slope. A series of five test "pockets" were then dug into the existing slope such that a face of each pocket paralleled the trench. This arrangement provided for increasing distance to the trench as the LAS was moved from pocket to pocket. (See Figure 4)
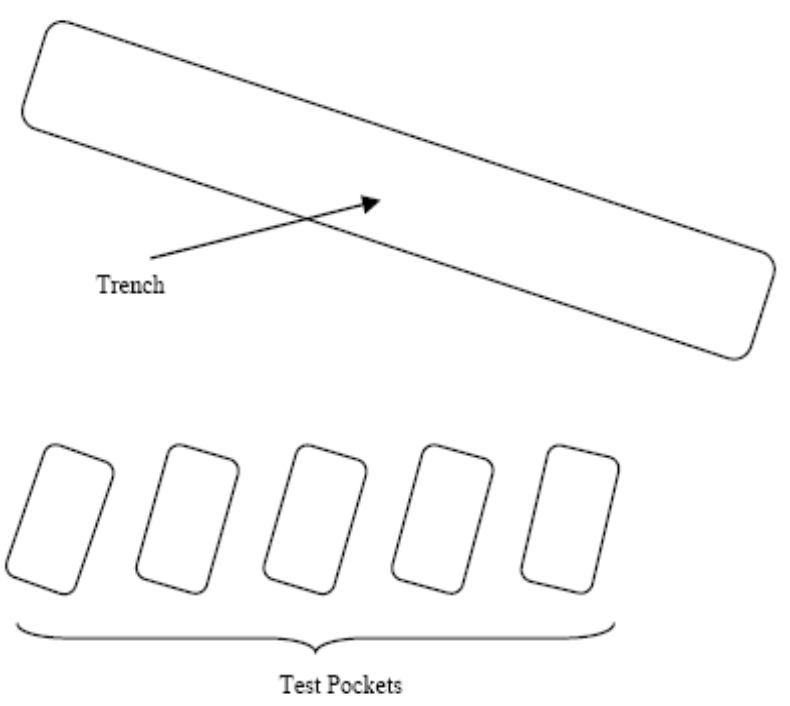

Figure 4. Rye Grass Flats 


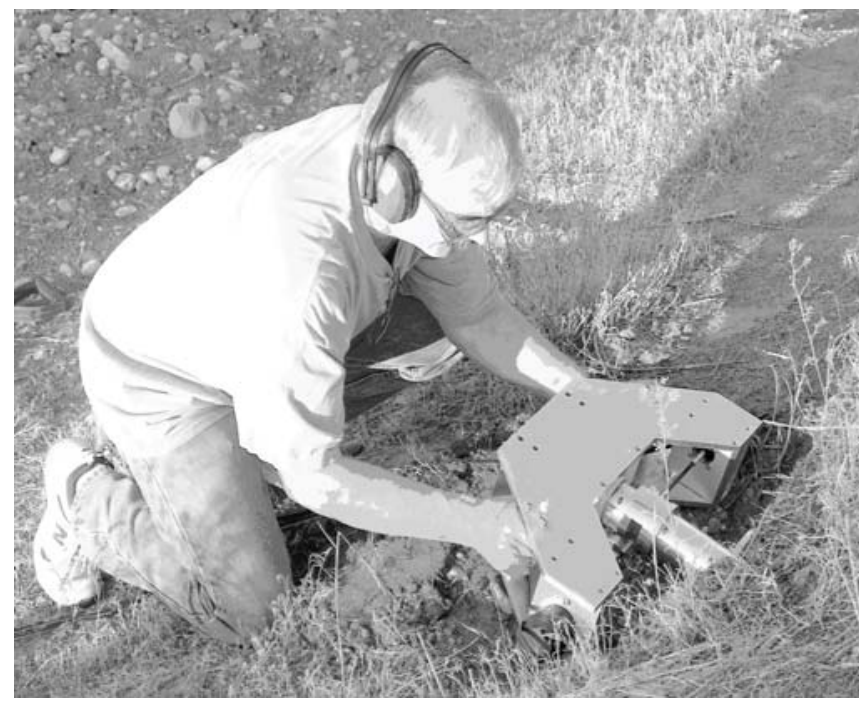

Figure 5. Operation of the Look-Ahead Sensor

The typical test procedure is as follows:

- $\quad$ Prepare a contact surface

- $\quad$ Set the power supply and control system for maximum voltage and sweep ramp parameters (we used 8 seconds up and 8 seconds down

- $\quad$ Place the tool against the digface surface, compress the LAS spring, and depress the trigger

- Hold the LAS stationary against the digface during the scan. See Figure 5

The LAS was initially configured for decreased range (balance weights installed). Consequently, initial testing did not include an attempt to detect the furthest void. Operators observed the following; easy operation, low noise levels, minimal power consumption, good data fidelity, and overall excellent tool performance. Some short-range setting operations (high frequency) resulted in excessive movement of the tool against the digface.

Balance weights were removed (more imbalance and higher force) for subsequent testing at the RGF area. The combination of higher operational frequencies coupled with the removal of balance weights was expected to make the unit more difficult to hold during operation. However, actual testing demonstrated that this was not as great an issue as predicted. Therefore, tests were conducted at the higher frequencies, with the higher imbalance, as well. Even at these less than ideal settings, operation of the LAS was manageable. The ease of LAS operation even at higher frequencies indicates that the introduction of additional imbalance especially when implemented with more spring preload can increase the detection range (thereby increasing device flexibility). Configured with the higher imbalance and increased spring preload signal fidelity improved. Operating the tool near the upper limits of frequency, still, occasionally resulted in undesired, excessive tool movement (bounce) against the digface. This bounce phenomenon is detectable by the operator and confirmed by the data. To control this bounce further increasing spring preload would be required.

Configuring the LAS for operation at higher frequencies by adding balance weights and more spring load will likely improve short-range sensitivity and thus would provide for enhanced operational capability. This may be a key consideration when evaluating and undertaking future designs. The only likely adverse operational issue with such a design, besides decreased range, may be a small increase in operator effort due to increased holding force required to compress the higher preloaded spring. However automatic adjusting imbalance is also a consideration.

\section{Continuing Effort - IO Upgrades}

Subsequent efforts were made to enhance user interface and data acquisition. The results of these changes are less complex programming for mathematical processing, and improved raw and processed data quality. Specifically, a new motor control program raises the frequency in discrete steps so that steady-state conditions are established at 140 (each), approximately $1 \mathrm{~Hz}$ increments for a predetermined number of complete cycles. Also, the steps are initiated at a user-defined starting point when the LAS is triggered, operation then continues to the user-defined highest frequency and is then terminated. Early testing indicated that ramp up and back down was redundant, so a single ramp was programmed. The short duration discrete steps with improved data quality, the advanced start point, and the scan time shortening one-way ramp, all improved the data quality while reducing scan time. (8 to 12 seconds)

The data acquisition programming works in concert with this new motor-control logic. Data collection starts and stops nearly instantaneously, and concurrently with the initiation and termination of each discrete step. This results in data "bins" that contain amplitudes from nearly single frequency operation. These bins are recorded, and each bin includes enough data to fully establish 6 to 10 cycles of response. These data bins may or may not receive mathematical filtering at the time of recording but each bin also retains records of operational data.

Following the scan, a program calculates the exact frequency of each steady state bin and then calculates the average root-mean-square amplitude represented by the sinusoidal data in each bin. Next, the data points are plotted as 140 points of amplitude vs. frequency. The plot of this information can often be immediately interpreted by an experienced operator as to whether a void is present. Additionally, with a few quick calculations using plotted information, an estimated distance to a target can be derived using the equations previously discussed.

With the data reduced to amplitude verses frequency, a simple program can calculate and display the distance to 
target. Unfortunately, insufficient development funds were available to fully derive the necessary algorithms to facilitate this final computational stage. Nevertheless, we are confident that further refinement of the combined data collection and mathematical interpretation methods will result in a user interface where results are quick, definitive, and accurate.

\section{Experimental Results of new programming}

Two additional series of tests were conducted with the improved programming. The first set of tests used a partially buried bunker with a known configuration geometry. The second sequence of tests were performed in a drastically different geology and target characteristics.

\section{Partially Buried Bunker Testing}

The first set of tests of the new LAS configuration were conducted inside (looking outward) of a partially buried bunker. The side of the bunker was an embankment with an 8.2 meter thickness (at its greatest thickness). In addition to scanning the bunker embankment, vertical scans into the ground provided the baseline "no-void" condition. The new data collection and motor control logic provided a reasonable finite-distance-to-target response for the embankment thickness for the horizontal scan, and an infinite distance in the vertical scan. The new programming of the power supply provided for a voltage ramp up from approximately $50 \mathrm{~Hz}$ to user-defined maximum, in this case $180 \mathrm{~Hz}$.

Figure 6 shows the root-mean-square (RMS) velocity amplitude profile resulting from this frequency sweep. Two traces are provided-one is for a known no-void condition and the other for a known void at $7 \mathrm{~m}(23 \mathrm{ft})$. During these tests we found that the battery voltage was dropping and not providing the power for the set max speed. Consequently a new data input was added to the motor control program that

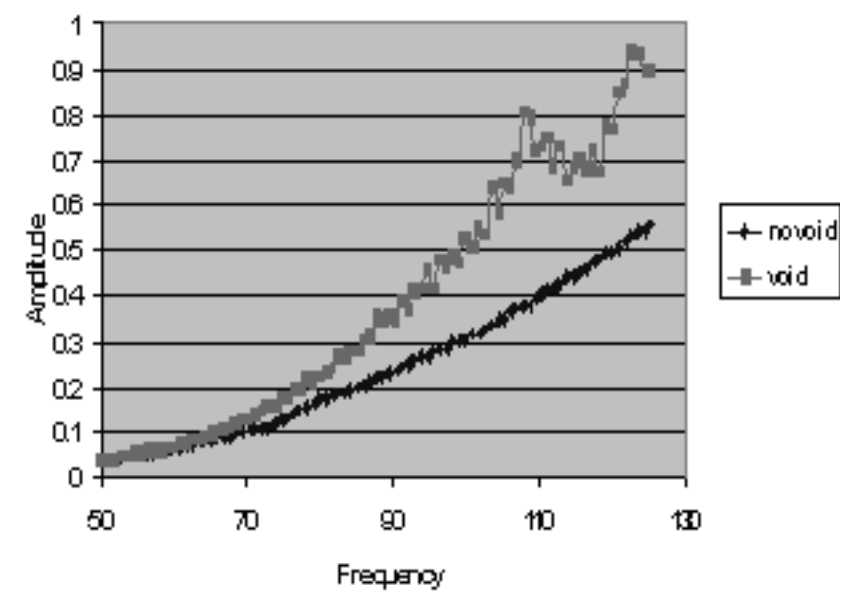

Figure 6. System Operational test raw data results for partially buried bunker accounted for battery voltage. This could be done automatically by using a feed back signal from the motors. With such a system not only would specific frequencies be defined in the programming but we would also obtain data of voltage (power) vs frequency. From this data, based on OV-PPL work, more media definition, perhaps even wave speed, will be available. Nevertheless, the lead-acid batteries were also replaced by lithium batteries for their uniform voltage during discharge and their much higher overall energy capacity.

\section{Testing in Alternate Field Conditions}

The second round of testing with the new IO system was conducted in an area offering very novel conditions. The target voids were usually steel-reinforced concrete lined, underground structures and occasionally steel lined structures. Also, the geology was mostly QuaternaryTertiary sedimentary rock rather than the top-soil encountered in a previous tests. Unfortunately, a controlled test environment was never available to truly test out the LAS capabilities.

\section{General Observations of the Testing}

The new lithium batteries performed well, executing over 40 experiments with minimal drain or voltage slump. It is entirely possible that a practiced operator may be able to sense the subsurface architecture just by running the LAS. A good example of this occurred during one of the tests. Operation of the LAS at one specific location resulted in a perceptible dwelling of the frequency ramp. Repeated operation at that location resulted in the same observation. It was subsequently found that these scans occurred directly above a sub-surface vertical vent line riser. It is speculated that the peculiar behavior was due to the establishment of an standing wave harmonic with destructive interference.

\section{Test Results for the Alternate Test Site}

This round of testing demonstrated good performance even in novel conditions. At locations where no target existed, a predictable and repeatable no void output curve was generated. This "negative" indication was consistent and did not depend on location. Also, the signal quality was excellent in the rocky environment with reinforced concrete targets. In addition to the detection ("positive") of known voids and rejection in all cases where there was no void, the presence of a void was recognizable regardless of whether the LAS was aimed exactly at the void. (Figure 7) The test results were not conclusive as to whether the signal strength vs. view factor were related, but there seemed to be a correlation. It is expected that view-factor and direction indications may be resolvable with experience. In tests performed at the INL, the amplitude of the negative for 
target and the positive for target did not differ greatly. The distinction between positive and negative detection was found in the shape of the amplitude vs. frequency curve, though normalizing the amplitude by dividing out frequency squared accented the differences. In these off-site tests, however, dividing out frequency to the first power was all that was necessary. The second division to normalize displacement amplitude was not of great value. (Figure 8)



Figure 7. Frequency vs. Displacement Amplitude for Baseline (2) and for various Horizontal Scans at varying angles

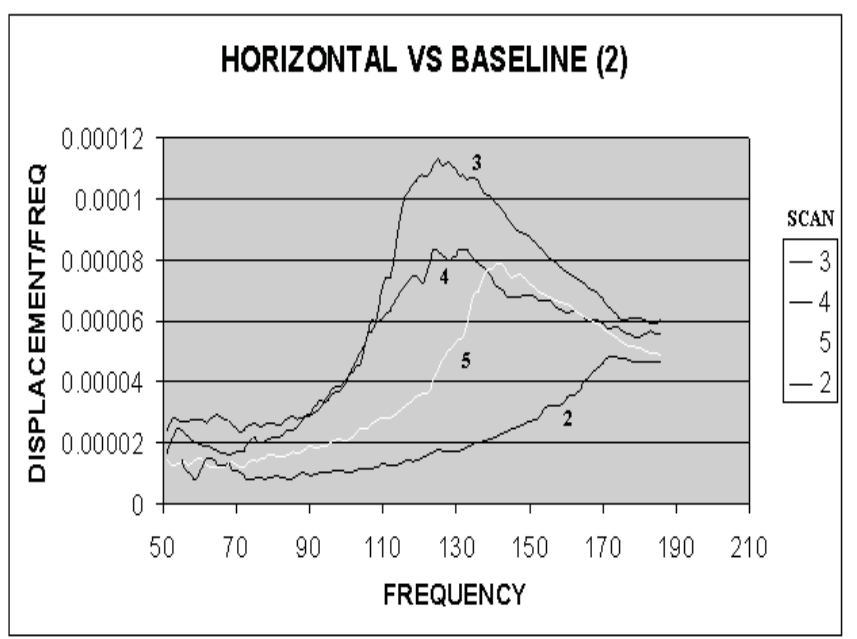

Figure 8. Normalized Frequency vs. Displacement Amplitude for Baseline and for various Horizontal Scans. (normalized by division of the frequency)

The data showed a consistently large difference in the amplitude between the negative and the positive scans. In figure 9, the range from 50 to $175 \mathrm{~Hz}$, scan 14 gives a trace indicating there was no void. Trace scan 20 was welldefined and right over a void. The difference between the two traces indicates how the LAS indicates a void. The significant rise in trace scan 20 over that of scan 14 is the key indicator. Trace scan 22 was at a location adjacent to scan 20, and also indicates a void. The two traces, scan 20 and scan 22 , demonstrate the slight difference in traces due to placing the LAS in adjacent locations.

\section{Interpretation}

In a fully deployable LAS, it is envisioned that the amplitude vs. frequency data will be fed to a decision algorithm that will determine the constructive/destructive frequency pattern and return a distance to the void. If no resonance frequency pattern is detected, then the algorithm will return a negative result indicating that no void is present. For a future system, it is unclear how far this process can go without human interpretation. But for the current system, the instant generation of the amplitude vs. frequency curve is useful to an experienced operator. While these field tests produced raw data and partially processed data that could be interpreted as to the presence of and distance to a void, it is still desirable to have a more userfriendly interface.

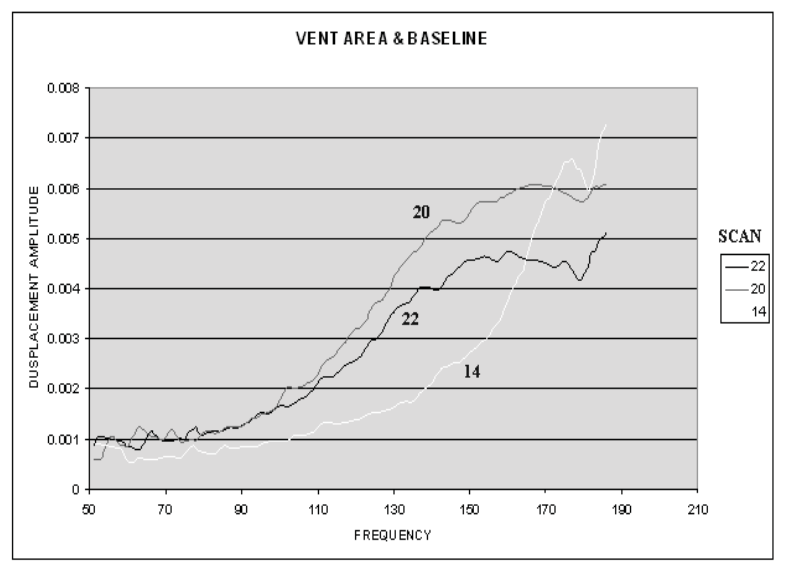

Figure 9. Frequency vs. Displacement Amplitude Curves for Baseline and the Vent Area Scans

\section{Conclusions}

The Idaho National Laboratory's Look Ahead Sensor prototype is capable of detecting, and measuring the distance to, an underground void through 4 meters to 23 meters of earth with a single fixed imbalance. Acoustic energy emitted by, and returned to the hand-held device, is sensed and recorded. The receipt can be processed and interpreted to indicate the presence or absence of a void and the distance to that void. The INL Look Ahead Sensor met all expectations of a functional prototype. It demonstrated proof of principle, field functional capabilities, and environmental adaptability, but further development is required to make it fully deployable. Further adaptations could also place it on autonomous ground vehicles. 


\section{ACKNOWLEDGMENTS}

I am grateful for the knowledge and talents provided by Dr. Stephen Novascone. His skills formed the basis of "driving point impedance" technology as used in the OV-PPL and the LAS. Dr. Novascone also provided the analytical support that created the baseline design details that would have otherwise required empirical derivation.

I am also thankful of the technical assistance provided by John Svoboda who's masterful electronics and programming skills made the motor control and data collection possible.

This manuscript has been authored by Battelle Energy Alliance, LLC under Contract No. DE-AC07-05ID14517 with the U.S. Department of Energy. The United States Government retains and the publisher, by accepting the article for publication, acknowledges that the United States Government retains a nonexclusive, paid-up, irrevocable, world-wide license to publish or reproduce the published form of this manuscript, or allow others to do so, for United States Government purposes.

\section{REFERENCES}

[1] S. R. Novascone and D. M. Weinberg. Frequency shift of a rotating mass-imbalance immersed in an acoustic fluid. Acoustical Society of America 118 (2) Aug, 2005

[2] J. H. Cole, The orbital vibrator a new tool for characterizing interwell reservoir space The Leading Edge 16, 281-283 1997 